\title{
Halogenated Hydrocarbon
}

National Cancer Institute

\section{Source}

National Cancer Institute. Halogenated Hydrocarbon. NCI Thesaurus. Code C554.

Compounds consisting of carbon, hydrogen, and one or more halogen. 